\title{
La distension intestinale pendant la chirurgie abdominale élective: doit-on bannir le protoxyde d'azote?
}

\begin{abstract}
Nous avons étudié la distension intestintale secondaire au protoxyde d'azote chez 20 pcitients devant subir une laparotomie pour chirurgie intestinale élective. Les patients ont été répartis de façon aléatoire en deux groupes. Cette répartition d́tait inconnue des chirurgiens. La technique aresthésique était identique chez tous les patients, exception faite de l'utilisation du protoxyde d" azote dans le Groupe II. $\mathrm{La} \mathrm{FlO}_{2}$ était maintenue à 0.4 dans les deux groupes. L'anesthésie était maintenate avec un mélange airloxygènefenfurane dans le Groupe $I$ et, dans le Groupe $I$, avec un mélange prosoxydeloxygènelenflurane. La curarisation était gardée optimale tout au long de l'intervention. Le perimètre abdominal el la circonférence de l'iléon terminal et du côlon transverse ont été mesurés au début es à la fin de l'intervention. Le chirargien devait grader la difficulté qu'il éprouvait à fermer la plaie abdominale d la fin de la chirurgie selon une échelie pré-établie. La durée de la chirurgie était semblable dans les deux groupes. Nous avons noté une distension statistiquement significative mais cliniquement non-appréciable de l'itéon terminal dans le Groupe II. Dans le Groupe I, la circonférence du côlon transverse a diminuè de façon significative, La difficulté à fermer l'abdomen fut de légère à nulle dans les deux groupes. Nous n'avons pas réussi à assacier de façon significative la présence de protoxyde d'azote avec une difficulté accrue à fermer la plaie abdominole. Nous concluons que, chez l'adulte, des concentrations de 60 pour cent de protoxyde d"azole ou moins peuvent être utilisées pour des chirurgies intestinales électives d' une durée moyenne d' environ 90 minutes sans crainte de causer une distension intestinale cliniquement significarive ou une difficulté accrue da fermer l'abdomen.
\end{abstract}

Mots clés

CHIRURGIE: chirurgie intestinale élective; $\mathrm{GAZ}$ ANESTHESIQUE: protoxyde d'aZote; COMPLICATION: distension intestinale.

Département d'Anesthésie-Réanimation, Hôpital MaisonneuveRosemont, Université de Mantréal, Montréal, Québec.

Pour correspondance: Docteur Jean-François Hardy, Département d'Anesthésiè-Réanimation, Hópital MaisonneuveRosemont, 5415, Boul. de l'Assommption, Montréal, P. Québec, H1T 2M4.

Une partie de ce travail a êté présentée au congrès de l'American Society of Anesthesiologists tenu à San Francisco en 1985.
Le problème de la distension intestinale secondaire à l'usage de protoxyde d'azote $\left(\mathrm{N}_{2} \mathrm{O}\right)$ en cours d'anesthésie est bien connu chez les animaux ${ }^{1,2}$ et les humains ${ }^{3}$ porteurs d'iléus ou d'obstruction intestinale. On a également noté le phénomène lorsque le transit intestinal est normal chez le poney ${ }^{4}$ et chez l'humain. ${ }^{5}$ Cette dernière étude faite au cours de chirurgic non-abdominale a montré une augmentation significative du périmètre abdomina! avec l'emploi du protoxyde d'azote pendant l'opération. Les auteurs concluaient que: "le fait d'éviter des concentrations élevées de protoxyde durant les cas de chirurgie abdominale peut faciliter la tâche du chirurgien, raccourcir la durée de l'intervention et ainsi être bénéfique pour le malade". 5

Nous avons done entrepris cette étude dans le but d'objectiver la distension intestinale secondaire au protoxyde d'azote lors de laparotomie pour une intervention élective au niveau de l'intestin. De plus, nous voulions vérifir si l'emploi du protoxyde d'azote dans ces cas est associé à une difficulté accrue à fermer la plaic abdominale.

\section{Mëthode}

L'étude a êté approuvée par Je comité d'éthique médicale de l'hôpital Maisonneuve-Rosemont. Les techniques anesthésiques utilisćes étant de pratique courante dans notre département, le comité n'a pas exigé de consentement écrit des patients.

Vingt patients, de statut physique I, II ou III selon la classification de l'ASA, devant subir une laparotomie pour résection intestinale ou colotomie ont été divisés en deux groupes égaux selon un mode aléatoire. Les patients ne devaient pas avoir de contre-indication à l'usage du protoxyde d'azote comme agent anesthesique. Aucun ne présentait d'iléus paralytique ou d'obstruction intestinale pré-opératoire.

En prémédication, 19 patients ont reçu une benzodiazépine (18 du lorazépam $0.03 \mathrm{mg} \cdot \mathrm{kg}^{-1}$, un du diazépam $\left.0.1 \mathrm{mg} \cdot \mathrm{kg}^{-1}\right)$ et un patient $\mathrm{n}^{\prime} \mathrm{a}$ rien reçu. Après préoxyténation, l'induction consistait en fentanyl $8 \mu \mathrm{g} \cdot \mathrm{kg}^{-1}$ DTC $0.5 \mathrm{mg} \cdot \mathrm{kg}^{-1}$, thiopental $2-5 \mathrm{mg} \cdot \mathrm{kg}^{-1}$ et succinyl- 
choline $1.5 \mathrm{mg} \cdot \mathrm{kg}^{-1}$. Les patients n'ont pas été ventilés au masque afin d'eviter toute distension gastrique. La trachée était ensuite canulée avec un tube endo-trachéal muni d'un ballonnet. La respiration était contrôlée mécaniquement pour maintenir une $\mathrm{PaCO}_{2}$ entre 35 et $40 \mathrm{mmHg}$. Les patients du Groupe I étaient ventilés avec un mélange d'air et d'oxygène et ceux du Groupe II avee un mélange de protoxyde d'azote et d'oxygène pour maintenir la $\mathrm{FIO}_{2}$ (mesurée dans la branche inspiratoire du circuit en cercle avec un analyseur d'oxygène Ohio modèle 401) à 0.4 dans les deux groupes. Tous reçurent de l'enflurane à une concentration suffisante pour maintenir les signes vitaux dans les limites de la normale. Les patients étaient curarisés avec du pancuronium et le degré de curarisation monitoré au "train de quatre" avec un neuro-stimulateur (Ohio -- Ministim MS-1) sur le trajet du nerf cubital. La profondeur du bloc était maintenue à une contraction visible ou moins des doigts tout au long de l'intervention. Une sonde naso-gastrique était mise en place ct le contenu de l'estomac aspiré, sa position étant vérifiée par l'écuipe chirurgicale. En fin d'intervention, les patients etaient décurarisés avec un mélange de néostigmine et de glycopyrrolate.

Toutes les mesures ont été réalisécs par la même équipe chirurgicale et anesthésique. Le choix alétoire de protoxyde d'azote ou air ćtait inconnu des chirurgiens et leur était caché pendant l'intervention. L'équipe anesthésique mesurait le périmètre abdominal du paticnt au niveau de J'ombilic en pré- et post-opératoire. L'équipe chirurgicale se servait de cordonnets pour marquer, à l'aide de pinces hémostatiques, l'hémi-circonférence de J'iléon terminal et du côlon transerse au début et à la fir de l'intervention. La longeur ainsi obtenue était ensuite mesurée par les anesthésistes, et était donc inconnue des chirurgiens. Le site de mesure devait être libre de toute pathologie intestinale. Le chirurgien devait évaluer si la distension intestinale causait une difficulté à fermer la plaie abdominale selon léchelle suivante: 0 - aucune difficulté, 1 difficulté légère, 2 - difficulté modérée, 3 - difficulté sévère, et 4 - impossibilité de fermer l'abdomen. On a de plus noté chez tous les patients la présence ou l'absence de perstaltisme au niveau de l'intestin grêle en fin d'intervention.

Les tests statistiques utilisés ont été le test exact de Fisher, le test " $t$ " de Student pour données appariées ou non-appariées, ainsi qu'une analyse de régression lineaire. Un " $\mathrm{p}$ " $<0.05$ a été considéré significatif.

\section{Resultats}

Il n'y avait pas de différence significative en ce qui a trait à 1'âge, au sexe ou au poids des patients. Les interventions chirurgicales étaient comparables pour les deux groupes (Tableaux I et II).
TABLEAU I Variables de personnes (moyenne \pm ecart type)

\begin{tabular}{|c|c|c|c|c|}
\hline & \multirow{2}{*}{$\begin{array}{l}\text { Age } \\
\text { (ans) }\end{array}$} & \multicolumn{2}{|c|}{ Sexe } & \multirow{2}{*}{$\begin{array}{l}\text { Poids } \\
\text { (kg) }\end{array}$} \\
\hline & & $F$ & $M$ & \\
\hline $\begin{array}{l}\text { Groupe I } \\
\text { (air) }\end{array}$ & $54.6 \pm 17.5$ & 3 & 7 & $77.0 \pm 13.3$ \\
\hline \multirow{2}{*}{$\begin{array}{l}\text { Groupe I] } \\
\left(\mathrm{N}_{2} \mathrm{O}\right)\end{array}$} & $57.4 \pm 16.9$ & 6 & 4 & $54.6 \pm 18.7$ \\
\hline & $p>0.5$ & \multicolumn{2}{|c|}{$\mathrm{p}=0.15$} & $p>0.1$ \\
\hline
\end{tabular}

La durée de la chirurgie ( 90 minutes environ) n'était pas statistiquement différente entre les deux groupes (Tableau III). La circonférence de l'iléon terminal chez les patients du Groupe I et la circonférence du côlon transverse chez les patients du Groupe II n'ont pas varié de façon significative entre le début et la fin de l'intervention (Tableau III).

Nous avons noté une distension significative de l'iléon terminal chez les patients du Groupe II, ainsi qu'une diminution statistiquement significative de la circonférence du côlon transverse chez les patients du Groupe I (Tableau III). Nous avons tenté d'établir une corrélation entre ces variations de circonférence et la durée de la chirurgie (Figure). L'expansion de l'ilean terminal chez les patients du Groupe II se faisait lentement (environ 45 pour cent en deux heures). Cette corrélation atteignait presque la significativité statistique $(p=0.055)$. Il $n^{\prime} y$ avait aucune corrélation entre la diminution de volume du côlon transverse chez les patients du Groupe I et la durée de la chirurgie.

Le périmètre abdominal n'a pas varié de façon significative (Tableau III). Le péristaltisme intestinal était présent en fin d'intervention chez tous les patients.

Cliniquement, l'utilisation du protoxyde d'azote n'a pas été associée à une distension intestinale perceptible

\section{TABLEAU II Type de chirurgie}

\section{Groupe I}

2 sigmoïdectomies

1 sigmoildectomie + colostomie

1 colostomie + sphinctéroplastie

3 hémicolectomies droites

2 abdomino-périnéales

1 iléostomie de Brooks

Groupe II

1 sigmoidectomic

2 sigmoidectomics + colostomies

1 sigmoïdectomie + colostomie + sphinctéraplastie

1 sigmoidectomie + sphinctéroplastie

2 húrmicolectomies droites

2 ahdomino-périnéales

1 "Pull through" de Steeve 
TABIEEAU III Variations des circonférences intestinales et du périmètre abdoninal (moyenne \pm ecart type)

\begin{tabular}{|c|c|c|c|c|c|c|c|}
\hline & \multirow{2}{*}{$\begin{array}{l}\text { Temps } \\
\text { chirurgical } \\
\text { (min) }\end{array}$} & \multicolumn{2}{|c|}{$\begin{array}{l}\text { Mesures des circonferences } \\
\text { de l'ileon remminol }\end{array}$} & \multicolumn{2}{|c|}{$\begin{array}{l}\text { Mesures des circunférences } \\
\text { du cblon transwerse }\end{array}$} & \multicolumn{2}{|c|}{$\begin{array}{l}\text { Mesture des varialions du } \\
\text { perimerre abdominal }\end{array}$} \\
\hline & & $\begin{array}{l}\text { Isitial } \\
(\mathrm{cm})\end{array}$ & $\begin{array}{l}\text { Final } \\
(\mathrm{cm})\end{array}$ & $\begin{array}{l}\text { Initial } \\
(\mathrm{cm})\end{array}$ & $\begin{array}{l}\text { Final } \\
\text { (cont) }\end{array}$ & $\begin{array}{l}\text { Initial } \\
\text { (cm) }\end{array}$ & $\begin{array}{l}\text { Final } \\
\text { (cm) }\end{array}$ \\
\hline $\begin{array}{l}\text { Groupe I } \\
\text { (air) }\end{array}$ & $96.9 \pm 50.4$ & $4.9 \pm 1.2$ & $5.2 \pm 1.3 \mathrm{NS}$ & $9.5 \pm 1.6$ & $8.0 \pm 1.7^{*}$ & $92.3 \pm 11.9$ & $94.3 \pm 11.4 \mathrm{NS}$ \\
\hline \multirow{2}{*}{$\begin{array}{l}\text { Groupe II } \\
\left(\mathrm{N}_{2} \mathrm{O}\right)\end{array}$} & $85.9 \pm 37.3$ & $4.5 \pm 1.2$ & $5.8 \pm 1.0 \dagger$ & $9.2 \pm 1.7$ & $9.3 \pm 2.0 \mathrm{NS}$ & $86.4 \pm 13.2$ & $86.9 \pm 12.6 \mathrm{NS}$ \\
\hline & NS & NS & & NS & & $N S$ & \\
\hline
\end{tabular}

NS: pas de différence significative entre les Groupes I et ll, ou entre la mesure initiale et finale.

$* p<0.05$.

$\dagger p<0.01$.

par les chirurgiens, ni responsable de difficultés lors de la fermeture de la paroi abdominale. En effet, la difficulté à fermer l'abdomen fut de nulle à légère dans les deux groupes (Tableau IV).

\section{Discussion}

Les gaz intestinaux, soit l'azote, l'hydrogène, le gaz carbonique et le méthane, sont en équilibre avec les gaz sanguins dans des conditions normales. Lorsque le patient est anesthésié avec du protoxyde d'azote et de l'oxygène, cet équilibre est rompu. Rapidement, la pression partielle de l'azote dass le sang devient nulle. Alors un nouvel équilibre tend à se faire. Le protoxyde d'azote diffuse vers les cavités libres et l'azote passe vers le sang. Le protoxyde étant 36 fois plus soluble que l'azote, il en résulte donc un transfert net de protoxyde vers la lumière intestinale. De plus, ce transfert provoque une dilution des gaz intraluminaux, abaissant leur pression particlle ct diminuant ainsi leur absorption par le sang. Donc, la distension est l'effet de la diffusion du protoxyde vers l'intestin et de la diminution de l'absorption des gaz intestinaux.

L'importance de l'augmentation du volume intraluminal est en relation avec 1) la quantité de gá présente initialement, 2) la concentration de protoxyde administrée,

TABLEAU IV Evaluation clinique de la difficulté de fermeture de la plaie abdominale

\begin{tabular}{lllll}
\hline \multicolumn{5}{l}{ Degré de difficuite } \\
\cline { 2 - 5 } & 0 & 1 & 2 & $3 \& 4$ \\
\hline Groupe I & 8 & 2 & 0 & 0 \\
Groupe II & 9 & 0 & 1 & 0 \\
& $* \mathrm{p}=0.39$ & & & \\
\hline
\end{tabular}

*Pour les fins du calcul statistique, les classes ont été restreintes à: aucune difftculté vs difficulté et comparés avec un test de Fisher. et, 3) la durée de l'exposition. La vitesse d'expansion dépendra de l'apport sanguin local. Théoriquement, le volume final sera un multiple ' $M$ ' du volume initial où:

$\mathrm{M}=\frac{100}{100-\% \mathrm{~N}_{2} \mathrm{O}},($ reference 6$)$,

en autant que les gaz intraluminaux ne diffusent pas vers le sang, que les parois de l'intestin soient parfaitement distensibles et que le segment intestinal soit clos et normalement vascularisé. $\mathrm{Si}$, compte tenu des petits nombres impliqués, l'on accepte la corrélation ( $r=0.63$, $p=0.055$ ) entre la variation de circonférence de l'iléon terminal et la durée de la chirurgie, l'expansion notée dans le Groupe II de cette étude correspond bien à la dynamique décrite par Eger en $1965 .{ }^{1}$ Le fait que cette expansion n'ait pas été remarquable (ou nuisible) pour les chirurgiens peut s'expliquer de deux façons. D'abord, il $s$ 'agissait de chirurgic électives et, avec un transit digestif normal et une préparation intestinale adéquate, l'intestin contenait une quantité minimale de gaz. Ensuite, la durée relativement brève de la chinurgie ne permettait pas une expansion maximale du tube digestif. Chez l'animal, celle-ci se fait au rythme approximatif de 70 pour cent en deux heures et 150 pour cent en quatre heures, l'expansion n'étant pas encore tout à fait complétée après ce temps. ${ }^{1}$

Expérimentalement, le colon et l'intestin grêle se distendent au mème rythme. ${ }^{\prime}$ Dans cette étude, nous avons observé une diminution de la circonférence du côlon transverse dans le Groupe I. L'absence de corrélation entre cette diminution et la durée de l'intervention nous porte à croire que cette variation de circonférence du côlon transverse n'est pas en relation avec 1'utilisation du protoxyde d'azote. Il est probable que ces changements de circonférence soient plus en rapport avec la chirurgie même si, théoriquement, la technique opératoire n'autorise aucune fuite de gaz au moment de l'anastomose des 


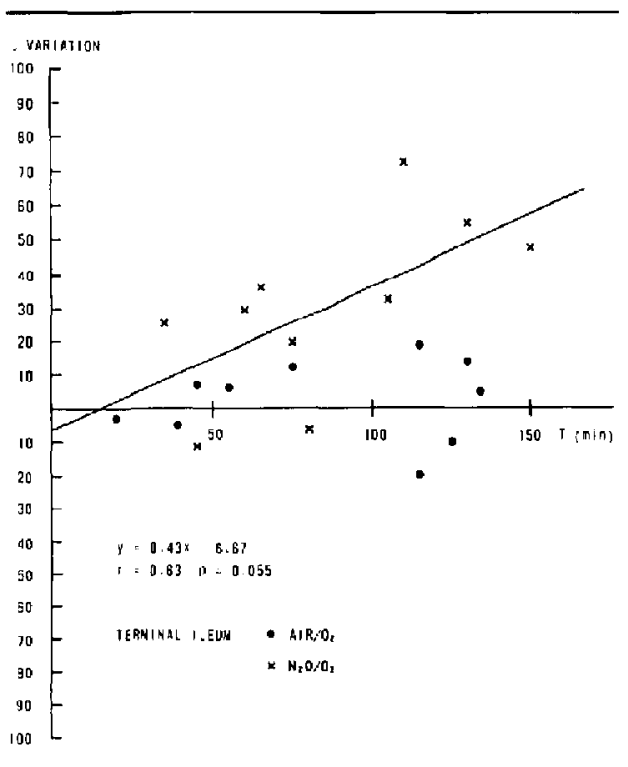

FIGURE Variations de la ciroonférence de l'iléon terminal en fonction de la durée de la chinurgie. La droite représente le résultat de l'analyse de tégression linéaire pour le groupe II $\left(\mathrm{N}_{2} \mathrm{O} / \mathrm{O}_{2}\right)$.

segments intestinaux. Nous ne sommes donc pas vraiment en mesure d'expliquer pourquoi il existe une différence significative entre ces deux groupes.

Nous n'avons pas trouvé de variation significative de la circonférence du périmètre abdominal, malgré les variations du périmètre intestinal mentionnées ci-haut. Le périmètre abdominal apparaît donc comme un indicateur peu sensible de la distension intestinale, du moins dans le contexte péri-opératoire décrit ici.

Depuis les études de Eger et coll. ${ }^{1,2,6,7}$ le protoxyde d'azote est certainement considéré par la plupart des anesthésistes comme étant contre-indiqué dans les cas d'obstruction intestinale, de peur d'aggraver la distension. De plus Eger suggère, mais cette hypothèse n'a jamais êté démontrée chez l'humain, que la distension intestinale secondaire au protoxyde d'azote pourrait être responsable de difficultés lors de la fermeture de la paroi abdominale lorsque la chirurgie est prolongee. ${ }^{1} \mathrm{Ce}$ concept a été élargi par Lewis ${ }^{5}$ à toutes les chirurgies abdominales électives dans l'espoir de rendre le travail du chirurgien plus facile. Toutefois il peut être indiqué, dans certains cas, d'utiliser le protoxyde d'azote afin, par exemple, de minimiser la dépression cardiovasculaire produite par les agents halogénés. ${ }^{7}$

Cette impression clinique d'une fermeture plus aisće de la paroi abdominale lorsque l'on évite le protoxyde (et que l'on utilise donc un agent volatil) pourrait être secondaire à un meilleur relächement de la paroi abdominale associé à l'utilisation des agents halogénés (en particulier l'enflurane ou l'isotlurane), surtout lorsque le bloc neuromusculaire, souvent non-monitoré, n'est pas optimal.

En conclusion, l'emploi de 60 pour cent de protoxyde d'azote pour des chirurgies intestinales électives de durée moyenne (environ 90 minutes) chez l'adulte ne semble avoir prolongé la durée de l'intervention et n'a pas été associé à une difficulté accrue à fermer la paroi abdominale malgré une discrète, bien que statistiquement significative, distension de l'iléon terminal. Dans ce contexte, l'emploi de concentrations de protoxyde d'azote de 60 pour cent ou moins n'apparaît donc pas être contreindiqué pour ce type de chirurgie.

\section{Remerciements}

Les auteurs tiennent à remercier le Dr Serge Dubé, chirurgien, pour sa collaboration et son assistance en salle d'opération, Mme Christiane Côté B.Sc, pour son aide technique, et Mme Louise Chartier pour la dactylographie du manuscrit.

\section{References}

1 Eger El H, Saidman $W$. Hazards of nitrous oxide anesthesia. Anesthesiology 1965; 26: 61-6.

2 Steffey EP, Johnson BH, Eger EI II, Howland, D Jr. Nitrous oxide: effect on accumulation rate and uptake of bowel gases. Anesth Analg 1979; 58: 405-8.

3 Foldes FF, Keper EF, Ship $A G$. Severe gastro-intestinal distension during nitrous oxide and oxygen anesthesia. JAMA 1965; 194: 224-6.

4 Moens $Y$, De Moor A. Diffusion of nitrous oxide into the intestinal lumen of ponies during halothane - nitrous oxide anesthesia. Am J Vet Res 1981; 42: 1751-3.

5 Lewis GBH. Intestinal distension during nitrous oxide anaesthesia. Can Anaesth Soc J 1975; 22: 200-1.

6 Eger EI II. Anesthetic uptake and action. Baltimorc, Williams et Wilkins Co., 1974, 171-3.

7 Hickey RF, Eger EI II. Circulatory pharmacology of inhaled anesthetics, chapitre 20 dans: Anesthesia. Edité par Miller, R.D., 2 ème édition, New-York, ChurchillLivingston 1986, pp. 649-66.

\section{Abstract}

High concentrations (70-80 per cent) of nitrous oxide $\left(\mathrm{N}_{2} \mathrm{O}\right)$ have been shown to cause distension of gas-comaining distensible structures such as the intestines and are, for this reason, avoided by some during elective abdominal operations on the $G I$ tract. We undertook this study to determine if $\mathrm{N}_{2} \mathrm{O}$ was 
responsible of a measurable change in intestinal diameter or of clinical difficuity in closing the abdomen in patients undergoing elective intestinal surgery of intermediate duration. Twenty patients scheduled for elective abdominal surgery were studied. Premedication and induction were idential in all patients. Maintenance of anesthesia in Group I was with enfurane in airloxygen, and fentanyl. Patients in Group II received enflurane in $\mathrm{N}_{2}$ Oloxygen, and fentanyl. $\mathrm{FrO}_{2}$ was 0.4 in both groups and monitored meuromuscular blockade with pancuronium was kept optistal throughout the operation. The surgeon was blinded as to the random distribution of patients to either group. Girth measurements at the level of the umbilicus were recorded before induction and after surgery. The circumferences of the terminal ileum and of the transierse colon were measured at the beginning and at the end of surgery. The surgeon was asked to rate the difficulty in closing the abdomen as $0=$ none, $I=$ slight, 2 = moderate, $3=$ severe, $4=$ impossible. Data were analysed using Student's paired t test, unpaired t test and Fisher's exact test. $P<0.05$ was considered statistically significant. There was na significam difference berween groups in age, sex distribution, weight and duration of surgery (approximately 90 minutes in both groups). Difficuly in closing the abdomen was, at most, slight and could not be associated with the use of $\mathrm{N}_{2} \mathrm{O}$. A statistically significant, but clinically unnoticeable, increase of the circumference of the terminal ileum was found when $\mathrm{N}_{2} \mathrm{O}$ was used. This increase was approximately 45 per cent in two hours. In Group I the circumference of the transverse colon decreased significantly. No other statistically significant differentce was found between groups. Peristaltic activity was present at the end of surgery in all patients. High concentrations of $\mathrm{N}_{2} \mathrm{O} \gg 70$ per centi are certainly considered contra-indicated by most anaesthetists in the presence of bowel obstruction, because of the fear of increasing intestinal distension. This cancept has been extended to elective abdominal surgery in the hope of making the surgeon's task easier. However, $\mathrm{N}_{2} \mathrm{O}$ may be useful to produce less cardiovascular depression at a given MAC level with imhalation agents. This study demonstrates that use of 60 per cent $\mathrm{N}_{2} \mathrm{O}$ did not result in clinically significant bowel distension. even if the intestines were manipulated and traumatized during surgery. Anaesthesia with volatile agents, especially enflurane and isofurone, may be associated with the impression that abdominal closure is easier if neuromuscular blockade is not montitored and kept optimal with $\mathrm{V}$ agens. We conclude that concentrations of 60 per cent $\mathrm{N}_{2} \mathrm{O}$ or less may be used for elective bowel surgery lasting approximately 90 minutes withour fear of causing clinically significan intestinal distension or difficulty in closing the abdomen. 